\title{
Angiopoietin-like protein 8 in early pregnancy improves the prediction of gestational diabetes
}

\author{
Yun Huang ${ }^{1}$ Xin Chen ${ }^{2} \cdot$ Xiaohong Chen ${ }^{1} \cdot$ Yu Feng ${ }^{1} \cdot$ Heming Guo $^{1} \cdot{\text { Sicheng } \mathrm{Li}^{1}}^{1}$ \\ Ting Dai ${ }^{1} \cdot$ Rong Jiang ${ }^{1} \cdot$ Xiaoyan Zhang ${ }^{1} \cdot$ Chen Fang $^{1} \cdot \mathrm{Ji} \mathrm{Hu}^{1}$
}

Received: 14 July 2017 / Accepted: 16 October 2017 / Published online: 22 November 2017

(C) Springer-Verlag GmbH Germany, part of Springer Nature 2017

\begin{abstract}
Aims/hypothesis Screening high-risk individuals for gestational diabetes mellitus (GDM) in early pregnancy conventionally relies on established maternal risk factors; however, the sensitivity and specificity of these factors are not satisfactory. The present study aimed to determine whether the concentration of angiopoietin-like protein 8 (ANGPTL8), either alone or combined with other risk factors in early pregnancy, could be used to predict subsequent GDM.

Methods From August 2015 to January 2016, 474 women receiving prenatal care at around 12-16 weeks of gestation were recruited into the study. ANGPTL8 levels were measured at the first prenatal visit. All the participants received a 75 g OGTT during weeks 24-28 of gestation.

Results ANGPTL8 levels in early pregnancy were considerably higher in women who developed GDM than those who maintained normal glucose tolerance ( $2822 \pm 938$ vs $2120 \pm$
\end{abstract}

Yun Huang and Xin Chen contributed equally to this study.

Electronic supplementary material The online version of this article (https://doi.org/10.1007/s00125-017-4505-y) contains peer-reviewed but unedited supplementary material, which is available to authorised users.

Chen Fang

afa9911@sina.com

$\mathrm{Ji} \mathrm{Hu}$

huji@suda.edu.cn

1 Department of Endocrinology, The Second Affiliated Hospital of Soochow University, 1055 Sanxiang Road, Suzhou, Jiangsu, People's Republic of China

2 Department of Gynaecology and Obstetrics, The Second Affiliated Hospital of Soochow University, Jiangsu, People's Republic of China
$1118 \mathrm{pg} / \mathrm{ml}$, respectively; $p<0.0001)$. Multivariable logistic regression revealed that ANGPTL8 levels were significantly associated with risk of GDM independent of conventional risk factors. In addition, women in the highest quartile of ANGPTL8 concentration had an 8.75-fold higher risk of developing GDM compared with women in the lowest quartile (OR8.75, 95\%CI 2.43, 31.58). More importantly, incorporating ANGPTL8 into the conventional prediction model significantly increased the AUC for prediction of GDM (0.772vs $0.725 ; p=0.019$ ).

Conclusions Our study suggests that ANGPTL8 levels in early pregnancy are significantly and independently associated with risk of GDM at 24-28 weeks of gestation. Combining ANGPTL8 levels with conventional risk factors could thus improve the prediction of GDM.

Keywords Angiopoietin-like protein 8 - Early pregnancy Gestational diabetes $\cdot$ Prediction

$\begin{array}{ll}\text { Abbreviations } \\ \text { ANGPTL8 } & \text { Angiopoietin-like protein } 8 \\ \text { ALT } & \text { Alanine aminotransferase } \\ \text { AST } & \text { Aspartate aminotransferase } \\ \text { DBP } & \text { Diastolic blood pressure } \\ \text { FPG } & \text { Fasting plasma glucose } \\ \text { GDM } & \text { Gestational diabetes mellitus } \\ \text { GGT } & \gamma \text {-Glutamyl transpeptidase } \\ \text { IADPSG } & \text { International Association of the Diabetes and } \\ & \text { Pregnancy Study Groups } \\ \text { PPG } & \text { Post-load plasma glucose } \\ \text { ROC } & \text { Receiver operator characteristic } \\ \text { SBP } & \text { Systolic blood pressure }\end{array}$




\section{Introduction}

The last decade witnessed a dramatic increase in the prevalence of gestational diabetes mellitus (GDM), especially after new diagnostic criteria from the International Association of the Diabetes and Pregnancy Study Groups (IADPSG) were adopted [1]. In 2013, a large-scale study applying the new criteria reported that the prevalence of GDM in China was $17.5 \%$ [2].GDM not only produces early complications, but can also have long-term adverse effects on both mothers and their offspring. A meta-analysis of studies examining women from 6 weeks to 28 years postpartum indicated that the cumulative incidence of postpartum diabetes ranged from $2.6 \%$ (6 weeks postpartum) to over $70 \%$ ( 28 years postpartum) [3], which implies that the majority of women with GDM would develop type 2 diabetes if the follow-up period was long enough. Additionally, maternal hyperglycaemia in pregnancy is independently associated with the offspring's risk of metabolic disorders including abnormal glucose tolerance, obesity and hypertension [4-6].

Given the great harm that GDM can do to mothers and their offspring, early prevention and detection are essential. Accumulated evidence has suggested that GDM can be prevented by lifestyle interventions started in early pregnancy in high-risk women [7-9], although some prospective studies have shown that GDM diagnosed before 12 weeks of gestation is associated with adverse pregnancy outcomes despite early testing and treatment $[10,11]$. There is no consensus on the diagnostic criteria for defining early GDM. It is recommended that women at high risk of hyperglycaemia are screened in early pregnancy [1]. Conventionally, early screening for GDM in pregnancy is largely dependent on established maternal risk factors, including pregnancy age, parity, family history of diabetes, history of polycystic ovarian syndrome and BMI before pregnancy. In addition to conventional risk factors, elevated C-reactive protein [12] and leptin levels [13] and decreased adiponectin levels [14] in the first trimester have been reported to be associated with a higher risk of subsequent GDM. However, the sensitivity and specificity of a single index for predicting GDM are not satisfactory $[15,16]$.

Angiopoietin-like protein 8 (ANGPTL8; also named hepatocellular carcinoma associated protein-TD26, refeeding induced fat and liver (RIFL), lipasin and betatrophin) is one of the members of ANGPTL family, implicated in lipid and glucose homeostasis [17-19]. ANGPTL8 levels are found to be higher in GDM than in normal pregnancy [20-22]. Therefore, it is important to determine whether ANGPTL8 levels in early pregnancy are related to subsequent risk of GDM. The aim of this study was to examine the ability of ANGPTL8 levels in early pregnancy to predict GDM, and to determine whether combining ANGPTL8 with conventional risk factors could enhance the predictive ability.

\section{Methods}

Population We conducted a preliminary study that recruited 100 pregnant women in early pregnancy, with 14 women eventually developing GDM. The mean ANGPTL8 levels of the two groups were $2079 \pm 1035$ and $3079 \pm 793 \mathrm{pg} / \mathrm{ml}$. Based on the results; we used PASS 11.0 software (www. ncss.com/download/pass/updates/pass11/) to calculate the sample size. With the level of significance set at 0.05 and the power at 0.95 , the calculated sample size was 352 . Assuming that $15 \%$ of participants would be lost to followup, the minimum sample size should therefore be 405 .

From August 2015 to January 2016, we prospectively and consecutively recruited 474 women who were receiving prenatal care around 12-16 weeks of gestation into the present study. Women with multiple pregnancies, pre-existing diabetes, pregestational hypertension and stillbirth were excluded from the analysis. Women who had a fasting plasma glucose (FPG) level $\geq 7.0 \mathrm{mmol} / \mathrm{l}$ at the first prenatal visit were diagnosed as having pre-existing diabetes and were excluded from the study. All participants were administered a $75 \mathrm{~g}$ OGTT at 24-28 weeks of gestation. Ethical approval was granted by the ethics committee of the Second Affiliated Hospital of Soochow University. Written informed consent was obtained from each participant before enrolment.

Clinical variables and definition Maternal information including age, parity, history of macrosomia and family history of diabetes were collected at the first prenatal visit around 1216 weeks of gestation. Body weight and height were measured in kilograms and metres, respectively, from which BMI was calculated. Fasting serum glucose, total cholesterol, triacylglycerol, aspartate aminotransferase (AST), alanine aminotransferase (ALT) and $\gamma$-glutamyl transpeptidase (GGT) were measured using an automated biochemical instrument (Cobas 8000; Roche, Basel, Switzerland). Serum ANGPTL8 levels were quantified with a commercially available ELISA kit (catalogue no. E11644h; Wuhan EIAab Science, Wuhan, China) according to the manufacturer's instructions.

Around 24-28 weeks of gestation, a 75 g OGTT was administered to all participants. After an overnight fast, venous blood samples were drawn at 0,1 and $2 \mathrm{~h}$ after the $75 \mathrm{~g}$ glucose load. GDM was diagnosed by $75 \mathrm{~g}$ OGTT according to the IADPSG criteria [1]. Participants were classified as having GDM if they met one or more of the following criteria: FPG $\geq 5.1 \mathrm{mmol} / \mathrm{l}, 1 \mathrm{~h}$ plasma glucose $\geq 10.0 \mathrm{mmol} / 1$ or $2 \mathrm{~h}$ plasma glucose $\geq 8.5 \mathrm{mmol} / 1$.

Statistical analysis Analyses were performed using SAS version $9.3 \mathrm{https}: / /$ support.sas.com/documentation/installcenter/ 93/index.html). Data were presented as mean $\pm \mathrm{SD}$, or median (interquartile range). Fasting serum triacylglycerol, ALT, AST and GGT were logarithmically transformed (base 
10) before the analyses, owing to a non-normal distribution. Comparisons between different groups were made using ANOVA or $\chi^{2}$ test. Univariable and multivariable logistic regressions were used to evaluate the associations between GDM and the variables measured at the first prenatal visit. The AUC of the receiver operator characteristic (ROC) curve was computed using the predicted probability of GDM and the true status of GDM for each participant. The optimal cut-off points to predict GDM were determined by Youden's index. Two-sided $p<0.05$ was considered as representing statistical significance.

\section{Results}

The general characteristics of women with normal glucose tolerance and those who developed GDM are shown in Table 1. A total of 474 pregnant women were included in the present study, 88 of whom developed GDM, giving a prevalence of $18.6 \%$. Women who developed GDM were older, were more likely to be hepatitis B surface antigen (HBsAg) positive and had higher BMI, GGT and FPG levels in early pregnancy, compared with those who did not develop GDM. In contrast, nulliparity, levels of triacylglycerol, total cholesterol, ALT and AST and blood pressure did not differ between the two groups. ANGPTL8 levels were significantly higher in women who developed GDM compared with those who did not $(2822 \pm 938$ vs. $2120 \pm 1118 \mathrm{pg} / \mathrm{ml} ; p<0.0001$; Fig. 1). At weeks 24-28 of gestation, as expected, women who developed GDM had significantly higher values of FPG, 1hpost-load plasma glucose (PPG) and 2hPPG, as well as BMI, compared with those who did not develop GDM. In contrast, the change in BMI from early pregnancy to the second trimester did not differ between the two groups.

Age, BMI, FPG, GGT, HBsAg positivity and ANGPTL8 were related to risk of GDM using the univariable logistic
Table 1 General characteristics of women of normal glucose tolerance and those who developed GDM

\begin{tabular}{|c|c|c|c|}
\hline Variable & Normal glucose tolerance $(n=386)$ & $\operatorname{GDM}(n=88)$ & $p$ value \\
\hline Age (years) & $28.1 \pm 3.7$ & $29.7 \pm 3.9$ & 0.0002 \\
\hline Nulliparity & $256(69.9)$ & $38(59.4)$ & 0.093 \\
\hline History of macrosomia $(n, \%)$ & $5(4.6)$ & $3(11.5)$ & 0.18 \\
\hline Family history of diabetes $(n, \%)$ & $7(1.91)$ & $3(4.69)$ & 0.17 \\
\hline \multicolumn{4}{|c|}{ Early pregnancy (gestational weeks $12-16$ ) } \\
\hline BMI $\left(\mathrm{kg} / \mathrm{m}^{2}\right)$ & $21.4 \pm 2.9$ & $22.6 \pm 3.4$ & 0.0037 \\
\hline FPG $(\mathrm{mmol} / \mathrm{l})$ & $4.54 \pm 0.35$ & $4.75 \pm 0.51$ & $<0.0001$ \\
\hline Total cholesterol (mmol/l) & $4.73 \pm 0.82$ & $4.80 \pm 0.87$ & 0.48 \\
\hline Triacylglycerol (mmol/l) & $1.24(1.00-1.53)$ & $1.33(1.05-1.79)$ & 0.18 \\
\hline $\operatorname{ALT}(\mathrm{U} / \mathrm{l})$ & $12(9-20)$ & $15(9-23)$ & 0.22 \\
\hline $\operatorname{AST}(\mathrm{U} / \mathrm{l})$ & $17(15-21)$ & $18(15-22)$ & 0.49 \\
\hline GGT (U/l) & $10(8-15)$ & $12(9-17)$ & 0.016 \\
\hline SBP $(\mathrm{mmHg})$ & $119 \pm 14$ & $122 \pm 13$ & 0.057 \\
\hline DBP (mmHg) & $70 \pm 9$ & $72 \pm 8$ & 0.14 \\
\hline HBsAg positive $(n, \%)$ & $8(2.7)$ & $6(8.7)$ & 0.02 \\
\hline ANGPTL8 (pg/ml) & $2120 \pm 1118$ & $2822 \pm 938$ & $<0.0001$ \\
\hline \multicolumn{4}{|c|}{ Second trimester (gestational weeks 24-28) } \\
\hline BMI $\left(\mathrm{kg} / \mathrm{m}^{2}\right)$ & $24.1 \pm 2.8$ & $25.3 \pm 3.5$ & 0.0017 \\
\hline $\mathrm{SBP}(\mathrm{mmHg})$ & $116 \pm 12$ & $117 \pm 17$ & 0.52 \\
\hline DBP (mmHg) & $68 \pm 8$ & $67 \pm 10$ & 0.71 \\
\hline$\Delta$ Body weight $(\mathrm{kg})$ & $6.93 \pm 3.54$ & $6.83 \pm 2.79$ & 0.83 \\
\hline$\Delta \mathrm{BMI}\left(\mathrm{kg} / \mathrm{m}^{2}\right)$ & $2.66 \pm 1.39$ & $2.63 \pm 1.04$ & 0.91 \\
\hline FPG (mmol/l) & $4.51 \pm 0.28$ & $5.18 \pm 0.70$ & $<0.0001$ \\
\hline 1hPPG (mmol/l) & $7.00 \pm 1.36$ & $9.18 \pm 1.91$ & $<0.0001$ \\
\hline $2 \mathrm{hPPG}(\mathrm{mmol} / \mathrm{l})$ & $6.28 \pm 0.96$ & $7.91 \pm 1.68$ & $<0.0001$ \\
\hline
\end{tabular}

Data are $n(\%)$, mean \pm SD or median (interquartile range)

Data were missing for family history of diabetes $(n=44)$, parity $(n=44)$, macrosomia $(n=1)$, BMI $(n=32)$, $\Delta$ body weight $(n=32)$, HBsAg $(n=109)$, total cholesterol $(n=35)$ or triacylglycerols $(n=35)$

$p$ values for differences between two groups were obtained by ANOVA or $\chi^{2}$ test

$\Delta$ Body weight, second-trimester body weight minus first-trimester body weight; $\Delta \mathrm{BMI}$, the second-trimester BMI minus the first-trimester BMI 


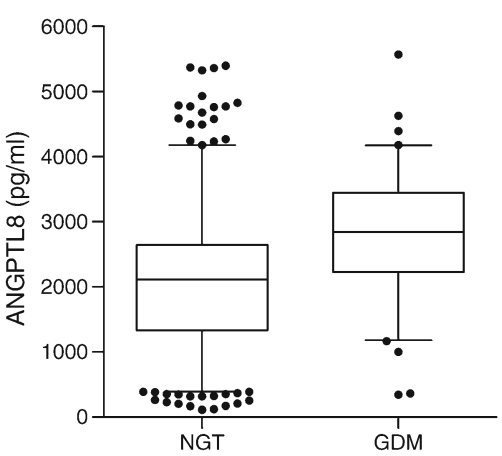

Fig. 1 ANGPTL8 levels in women with normal glucose tolerance (NGT) and women who developed GDM. ANGPTL8 levels were significantly higher in women who developed GDM than in those who did not $(p<0.0001)$

regression model (Table 2). After including these variables in the same model, age, FPG and ANGPTL8 level were still independently associated with risk of GDM, while BMI, GGT and HBsAg positivity were no longer related (Table 2).

The relationship between ANGPTL8 level and risk of GDM was further explored in different logistic regression models. The total population was divided into four groups according to ANGPTL8 level. Compared with a reference group of women in the lowest quartile of ANGPTL8 $(<1454 \mathrm{pg} / \mathrm{ml})$, women in quartile $3(2201-2870 \mathrm{pg} / \mathrm{ml})$ and quartile $4(>2870 \mathrm{pg} / \mathrm{ml})$ had a substantially higher risk of GDM (Table 3). After adjusting forage, parity, history of macrosomia, family history of diabetes, BMI, GGT, HBsAg, and FPG in early pregnancy, women in quartiles 3 and 4 still had a 4.92-fold (OR4.92, 95\%CI 1.33, 18.21) and 8.75-fold (OR8.75, 95\%CI 2.43, 31.58), respectively, higher risk of developing GDM.

To explore the predictive ability of conventional risk factors and ANGPTL8 level in early pregnancy, ROC curve analyses were performed (Fig. 2). The AUC for ANGPTL8 was 0.706 and was significantly greater than those for BMI, positive HBsAg and GGT $(p=0.028, p<0.0001$ and $p=0.015$, respectively; see electronic supplementary material [ESM] Table 1). Although the AUC of ANGPTL8 was also greater than those forage and FPG; the difference did not reach statistical significance. The optimal cut-off point of ANGPTL8 for predicting GDM was $2792.33 \mathrm{pg} / \mathrm{ml}$, with a sensitivity of $54.55 \%$, specificity of $79.27 \%$, positive predictive value of $37.5 \%$ and negative predictive value of $88.4 \%$. Prediction models incorporating clinical and biochemical risk factors were further analysed. In model 1 , the conventional risk factors of age, parity, family history of diabetes, history of macrosomia, and BMI, GGT, HBsAg and FPG in early pregnancy were included. The AUC for the conventional model was 0.725 (95\% CI $0.651,0.799)$. Introducing ANGPTL8 into the model statistically significantly increased the AUC to 0.772 (95\% CI $0.703,0.841 ; p=0.019)$.

\section{Discussion}

Our study showed that ANGPTL8 levels in early pregnancy were significantly associated with risk of subsequent GDM in weeks 24-28 of gestation, independently of conventional risk factors including maternal age, BMI, GGT, positive HBsAg and FPG levels in early pregnancy. In addition, ANGPTL8 had a greater AUC for predicting GDM than maternal BMI, GGT and positive HBsAg. More importantly, our results suggested that incorporating ANGPTL8 into the prediction model including conventional risk factors could significantly improve the ability to predict future GDM.

A75g OGTT performed during the late second trimester is currently regarded as the gold standard of GDM diagnosis, which may expose the infant to adverse intrauterine circumstances for too long. Hence there is a definite need to predict and detect GDM earlier in pregnancy in order to limit the harm that hyperglycaemia can do to the mother and her offspring. The performance of screening strategies based on conventional risk factors has been examined in many studies, but the sensitivity and specificity have not been shown to be satisfactory
Table 2 The risk factors associated with the incident GDM in early pregnancy

\begin{tabular}{|c|c|c|c|c|}
\hline \multirow[t]{2}{*}{ Variable } & \multicolumn{2}{|l|}{ Univariable } & \multicolumn{2}{|l|}{ Multivariable } \\
\hline & OR $(95 \% \mathrm{CI})$ & $p$ value & OR $(95 \% \mathrm{CI})$ & $p$ value \\
\hline Age (years) & $1.50(1.20,1.88)$ & 0.0003 & $1.74(1.28,2.39)$ & 0.0005 \\
\hline BMI $\left(\mathrm{kg} / \mathrm{m}^{2}\right)$ & $1.42(1.11,1.82)$ & 0.0046 & $1.13(0.83,1.54)$ & 0.43 \\
\hline FPG (mmol/l) & $1.70(1.34,2.16)$ & $<0.0001$ & $1.64(1.19,2.31)$ & 0.0032 \\
\hline $\log _{10}$ GGT $(\mathrm{mmol} / \mathrm{l})$ & $1.32(1.05,1.67)$ & 0.017 & $1.35(0.97,1.89)$ & 0.076 \\
\hline HBsAg positive (yes $=1$, no $=0$ ) & $3.43(1.10,10.20)$ & 0.027 & $1.33(0.22,5.77)$ & 0.88 \\
\hline ANGPTL8 (pg/ml) & $1.87(1.48,2.39)$ & $<0.0001$ & $1.88(1.37,2.63)$ & 0.0001 \\
\hline
\end{tabular}

ORs indicate the relative increased risk of GDM with each change in SD (for age, BMI, FPG, $\log _{10}$ GGT and ANGPTL8), or for a positive HBsAg. In multivariable regression analysis, these six variables were included in the same model 
Table 3 The association between ANGPTL8 levels in early pregnancy and the risk of GDM

\begin{tabular}{llllll} 
Variable & $\begin{array}{l}\text { Quartile 1 } \\
\text { Reference }\end{array}$ & OR $(95 \% \mathrm{CI})$ & $\begin{array}{l}\text { Quartile 3 }(2201-2870 \mathrm{pg} / \mathrm{ml}) \\
\text { OR }(95 \% \mathrm{CI})\end{array}$ & $\begin{array}{l}\text { Quartile } 4(>2870 \mathrm{pg} / \mathrm{ml}) \\
\text { OR }(95 \% \mathrm{CI})\end{array}$ \\
\hline Model 1 & 1.00 & $2.08(0.81,5.34)$ & $4.18(1.73,10.10)$ & $8.68(3.71,20.36)$ & $<.21 \mathrm{value}$ for trend \\
Model 2 & 1.00 & $1.40(0.44,4.48)$ & $3.80(1.34,10.79)$ & $8.21(2.98,22.58)$ & $<0.0001$ \\
Model 3 & 1.00 & $1.53(0.37,6.44)$ & $4.92(1.33,18.21)$ & $8.75(2.43,31.58)$ & $<0.0001$ \\
\hline
\end{tabular}

Model 1, unadjusted

Model 2, adjusted for age, parity, history of macrosomia, family history of diabetes and BMI in early pregnancy

Model 3, adjusted for age, parity, history of macrosomia, family history of diabetes, BMI, GGT, HBsAg, and FPG in early pregnancy

$p$ values were calculated from the logistic regression models

$[12,13,23,24]$. Among conventional risk factors, FPG is most commonly measured in early pregnancy. In a large Chinese population, FPG levels at the first prenatal visit were found to be positively associated with risk of GDM diagnosed at 2428 weeks [25]. Nevertheless, other studies have reported that FPG has poor sensitivity and specificity as a screening test in early pregnancy [23]. In our study, FPG in early pregnancy was independently associated with incidence of GDM. However, in line with the previous studies, the ability to predict GDM using FPG alone is limited, with an AUC of 0.646. Although many studies have demonstrated that increasing maternal BMI is a significant risk factor for the development of GDM [26, 27], a large sample study concluded that there was inadequate evidence to support the use of BMI alone to screen for GDM in early pregnancy [24]. In accordance with that study, we found that BMI in early pregnancy was inferior to FPG and ANGPTL8 in predicting GDM.

As the predictive performance of conventional risk factors to predict GDM is limited, more attention was paid here to other biomarkers. ANGPTL family members have been shown to play a major role in obesity and metabolic diseases $[28,29]$. It is well recognised that ANGPTL8 can act as a lipid regulator by inhibiting lipoprotein lipase activity, either directly or indirectly by promoting cleavage of ANGPTL3 [30, 31]. A recent animal study suggested that inhibition of ANGPTL8 might provide a new therapy for the treatment of dyslipidaemia, with beneficial effects on body weight [32]. Clinical epidemiological studies have provided abundant evidence regarding the associations between ANGPTL8 and metabolic diseases including diabetes [19, 33], hypertension [34], dyslipidaemia [17, 18] and the metabolic syndrome [35], as well as GDM [20-22]. In order to exclude the potential effect of blood pressure on ANGPTL8, we excluded participants with prehypertension (high normal blood pressure: systolic blood pressure [SBP] 120-139 $\mathrm{mmHg}$ or diastolic blood pressure [DBP] 80-89 $\mathrm{mmHg}$ ) from the present study. In case-control studies, participants who developed GDM had significantly higher levels of ANGPTL8 than healthy pregnant women [20,22]. Although ANGPTL8 levels change dramatically during pregnancy, no study has evaluated the role of
ANGPTL8 in early pregnancy in predicting GDM. To the best of our knowledge, our study has reported for the first time that ANGPTL8 levels in early pregnancy are positively and significantly associated with risk of subsequent GDM.

Some researchers argue that relying solely on conventional risk factors is unsatisfactory for predicting GDM; combining new biomarkers into a conventional predictive model may improve the prediction of GDM [36, 37]. Maitland and colleagues reported that introducing adiponectin, measured early in the second trimester, into the model based on routinely measured clinical factors significantly increased predictive ability [36]. Our study demonstrated that ANGPTL8, by itself, showed poor sensitivity for later GDM; however, adding ANGPTL8 to the model based on conventional risk factors in early pregnancy increased the ability to predict GDM.

The potential pathophysiological mechanism linking ANGPTL8 to GDM is not established. Ebert et al found that, during normal pregnancy, maternal ANGPTL8 levels were significantly higher than in the non-pregnant state, and they decreased in postpartum period [20]. The dynamic change in ANGPTL8 levels indicated that ANGPTL8 might participate in maintaining normal pregnancy. Moreover, ANGPTL8 levels have been found to be higher in umbilical cord blood than maternal serum [21], which suggests a potential role of ANGPTL8 in fetal growth and development. ANGPTL8 might thus offer us new insights into the physiology of normal pregnancy and the pathophysiology of GDM.

Some limitations of the present study should be noted. First, at the first prenatal visit, we did not perform a $75 \mathrm{~g}$ OGTT or measure $\mathrm{HbA}_{1 \mathrm{c}}$; although we excluded women with an FPG $\geq 7.0 \mathrm{mmol} / \mathrm{l}$, women with higher PPG but normal FPG levels might be misclassified as having normal glucose regulation. Second, the study was also limited by the lack of data on insulin levels; therefore we were unable to assess whether the ability of ANGPTL8 to predict GDM was mediated by insulin resistance or whether ANGPTL8 was merely a biomarker of insulin resistance. Although some researchers have found that ANGPTL8 can promote the suppression of key enzymes involved in gluconeogenic pathways and 

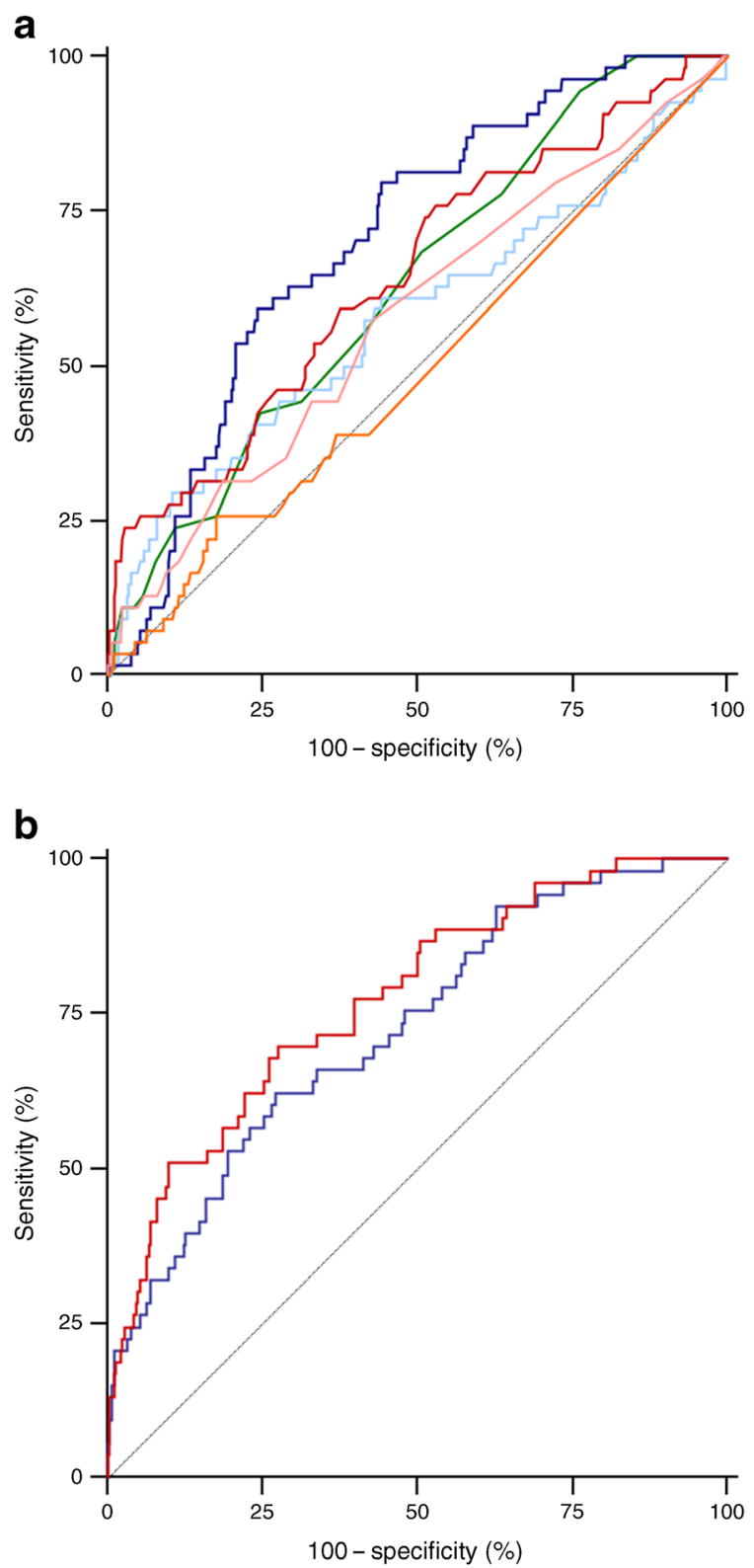

Fig. 2 ROC curves comparing the potential of different variables in early pregnancy to predict GDM. (a) The prediction of individual GDM variables. Dark blue, ANGPTL8; green, age; red, FPG; light blue, BMI; pink, GGT; orange, HBsAg. (b) Predictive ability of different models of GDM. Model 1 (blue), conventional model including age, parity, family history of diabetes, history of macrosomia and BMI, GGT, HBsAg and FPG in early pregnancy. Model 2 (red), model 1 plus ANGPTL8

improving insulin resistance [38], some clinical studies have demonstrated that the associations between ANGPTL8 levels and metabolic diseases were independent of insulin resistance $[18,35,39]$. Consistent with the studies performed in the general population, the association between ANGPTL8 and GDM still persisted after controlling for HOMA-IR in pregnant women [40]. Third, information on diet and physical activity was also lacking, and therefore we were not able to evaluate the effects of lifestyle change on risk of GDM.
Finally, although the ELISA kit for ANGPTL8 is now commercially available and inexpensive, the normal range of ANGPTL8 levels should be defined for future clinical application, so a large population sample is needed to do this.

In conclusion, the present study suggested that ANGPTL8 levels in early pregnancy were significantly and independently predictive of GDM in weeks 24-28 of gestation, and that combining ANGPTL8 levels with conventional risk factors for GDM could improve predictive performance.

Acknowledgements We are grateful to all the pregnant women who participated in this study.

Data availability The datasets generated during and/or analysed during the current study are available from the corresponding author on reasonable request.

Funding The work was supported by grants from the National Natural Science Foundation of China $(81471041,81502865,8160030570)$, the Natural Science Foundation of Jiangsu Province (BK20130276) and the Foundation of Jiangsu Provincial Commission of Health and Family Planning (Q201504).

Duality of interest The authors declare that there is no duality of interest associated with this manuscript.

Author contribution $\mathrm{YH}, \mathrm{XC}, \mathrm{CF}$ and $\mathrm{JH}$ conceived and designed the study. YH and XC analysed the data. XHC, YF, HMG, SCL, TD, RJ and $X Y Z$ contributed to acquisition of the data. YH, XC, HMG, SCL, TD, RJ and $\mathrm{XYZ}$ drafted the article. $\mathrm{XHC}, \mathrm{YF}, \mathrm{CF}$ and $\mathrm{JH}$ revised the article critically for important intellectual content. All authors approved of the final version to be published. JH is responsible for the integrity of the work as a whole.

\section{References}

1. International Association of Diabetes and Pregnancy Study Groups Consensus Panel, Metzger BE, Gabbe SG, Persson B et al (2010) International Association of Diabetes and Pregnancy Study Groups recommendations on the diagnosis and classification of hyperglycemia in pregnancy. Diabetes Care 33:676-682

2. Zhu W, Yang H, Wei Y et al (2015) Comparing the diagnostic criteria for gestational diabetes mellitus of World Health Organization 2013 with 1999 in Chinese population. Chin Med J 128:125-127

3. Kim C, Newton KM, Knopp RH (2002) Gestational diabetes and the incidence of type 2 diabetes: a systematic review. Diabetes Care 25:1862-1868

4. Damm P (2009) Future risk of diabetes in mother and child after gestational diabetes mellitus. Int J Gynaecol Obstet 104(Suppl 1): S25-S26

5. Hillier TA, Pedula KL, Schmidt MM, Mullen JA, Charles MA, Pettitt DJ (2007) Childhood obesity and metabolic imprinting: the ongoing effects of maternal hyperglycemia. Diabetes Care 30: 2287-2292

6. Boney CM, Verma A, Tucker R, Vohr BR (2005) Metabolic syndrome in childhood: association with birth weight, maternal obesity, and gestational diabetes mellitus. Pediatrics 115:e290-e296 
7. Zhang C, Tobias DK, Chavarro JE et al (2014) Adherence to healthy lifestyle and risk of gestational diabetes mellitus: prospective cohort study. BMJ 349:g5450

8. Koivusalo SB, Rönö K, Klemetti MM et al (2016) Gestational diabetes mellitus can be prevented by lifestyle intervention: the Finnish Gestational Diabetes Prevention Study (RADIEL): a randomized controlled trial. Diabetes Care 39:24-30

9. Wang C, Wei Y, Zhang X et al (2017) A randomized clinical trial of exercise during pregnancy to prevent gestational diabetes mellitus and improve pregnancy outcome in overweight and obese pregnant women. Am J Obstet Gynecol 216:340-351

10. Sweeting AN, Ross GP, Hyett J, Molyneaux L et al (2016) Gestational diabetes mellitus in early pregnancy: evidence for poor pregnancy outcomes despite treatment. Diabetes Care 39:75-81

11. Bartha JL, Martinez-Del-Fresno P, Comino-Delgado R (2000) Gestational diabetes mellitus diagnosed during early pregnancy. Am J Obstet Gynecol 182:346-350

12. Qiu C, Sorensen TK, Luthy DA, Williams MA (2004) A prospective study of maternal serum C-reactive protein (CRP) concentrations and risk of gestational diabetes mellitus. Paediatr Perinat Epidemiol 18:377-384

13. Qiu C, Williams MA, Vadachkoria S, Frederick IO, Luthy DA (2004) Increased maternal plasma leptin in early pregnancy and risk of gestational diabetes mellitus. Obstet Gynecol 103:519-525

14. Iliodromiti S, Sassarini J, Kelsey TW, Lindsay RS, Sattar N, Nelson SM (2016) Accuracy of circulating adiponectin for predicting gestational diabetes: a systematic review and meta-analysis. Diabetologia 59:692-699

15. Naylor CD, Sermer M, Chen E, Farine D (1997) Selective screening for gestational diabetes mellitus. Toronto trihospital gestational diabetes project investigators. N Engl J Med 337:1591-1596

16. Cosson E, Benchimol M, Carbillon L et al (2006) Universal rather than selective screening for gestational diabetes mellitus may improve fetal outcomes. Diabete Metab 32:140-146

17. Gómez-Ambrosi J, Pascual-Corrales E, Catalán V et al (2016) Altered concentrations in dyslipidemia evidence a role for ANGPTL8/betatrophin in lipid metabolism in humans. J Clin Endocrinol Metab 101:3803-3811

18. Fenzl A, Itariu BK, Kosi L et al (2014) Circulating betatrophin correlates with atherogenic lipid profiles but not with glucose and insulin levels in insulin-resistant individuals. Diabetologia 57: 1204-1208

19. Chen X, Lu P, He W et al (2015) Circulating betatrophin levels are increased in patients with type 2 diabetes and associated with insulin resistance. J Clin Endocrinol Metab 100:E96-E100

20. Ebert T, Kralisch S, Wurst U et al (2015) Betatrophin levels are increased in women with gestational diabetes mellitus compared to healthy pregnant controls. Eur J Endocrinol 173:1-7

21. Wawrusiewicz-Kurylonek N, Telejko B, Kuzmicki M et al (2015) Increased maternal and cord blood betatrophin in gestational diabetes. PLoS One 10:e131171

22. Martinez-Perez B, Ejarque M, Gutierrez C et al (2016) Angiopoietin-like protein 8 (ANGPTL8) in pregnancy: a brown adipose tissue-derived endocrine factor with a potential role in fetal growth. Transl Res 178:1-12

23. Yeral MI, Ozgu-Erdinc AS, Uygur D, Seckin KD, Karsli MF, Danisman AN (2014) Prediction of gestational diabetes mellitus in the first trimester, comparison of fasting plasma glucose, two- step and one-step methods: a prospective randomized controlled trial. Endocrine 46:512-518

24. Meek CL, Murphy HR, Simmons D (2016) Random plasma glucose in early pregnancy is a better predictor of gestational diabetes diagnosis than maternal obesity. Diabetologia 59:445-452

25. Zhu WW, Yang HX, Wei YM et al (2013) Evaluation of the value of fasting plasma glucose in the first prenatal visit to diagnose gestational diabetes mellitus in china. Diabetes Care 36:586-590

26. Martin KE, Grivell RM, Yelland LN, Dodd JM (2015) The influence of maternal BMI and gestational diabetes on pregnancy outcome. Diabetes Res Clin Pract 108:508-513

27. Li P, Yin Y, Lin S et al (2016) Utility of pregestational body mass index and initial fasting plasma glucose in predicting gestational diabetes mellitus. Am J Med Sci 351:420-425

28. Li Y, Teng C (2014) Angiopoietin-like proteins 3, 4 and 8: regulating lipid metabolism and providing new hope for metabolic syndrome. J Drug Target 22:679-687

29. Santulli G (2014) Angiopoietin-like proteins: a comprehensive look. Front Endocrinol (Lausanne) 5:4

30. Quagliarini F, Wang Y, Kozlitina J et al (2012) Atypical angiopoietin-like protein that regulates ANGPTL3. Proc Natl Acad Sci U S A 109:19751-19756

31. Zhang R (2012) Lipasin, a novel nutritionally-regulated liverenriched factor that regulates serum triglyceride levels. Biochem Biophys Res Commun 424:786-792

32. Gusarova V, Banfi S, Alexa-Braun CA et al (2017) ANGPTL8 blockade with a monoclonal antibody promotes triglyceride clearance, energy expenditure, and weight loss in mice. Endocrinology 158:1252-1259

33. Ebert T, Kralisch S, Hoffmann A et al (2014) Circulating angiopoietin-like protein 8 is independently associated with fasting plasma glucose and type 2 diabetes mellitus. J Clin Endocrinol Metab 99:E2510-E2517

34. Al-Daghri NM, Rahman S, Sabico S et al (2016) Circulating betatrophin in healthy control and type 2 diabetic subjects and its association with metabolic parameters. J Diabetes Complicat 30: $1321-1325$

35. Wang H, Lai Y, Han C et al (2016) The effects of serum ANGPTL8/ betatrophin on the risk of developing the metabolic syndrome- a prospective study. Sci Rep 6:28431

36. Maitland RA, Seed PT, Briley AL et al (2014) UPBEAT trial consortium. Prediction of gestational diabetes in obese pregnant women from the UK Pregnancies Better Eating and Activity (UPBEAT) pilot trial. Diabet Med 31:963-970

37. Savvidou M, Nelson SM, Makgoba M, Messow CM, Sattar N, Nicolaides K (2010) First-trimester prediction of gestational diabetes mellitus: examining the potential of combining maternal characteristics and laboratory measures. Diabetes 59:3017-3022

38. Rong Guo X, Li Wang X, Chen Y et al (2016) ANGPTL8/ betatrophin alleviates insulin resistance via the Akt-GSK3 $\beta$ or Akt-FoxO1 pathway in HepG2 cells. Exp Cell Res 345:158-167

39. Abu-Farha M, Abubaker J, Al-Khairi I et al (2015) Higher plasma betatrophin/ANGPTL8 level in type 2 diabetes subjects does not correlate with blood glucose or insulin resistance. Sci Rep 5:10949

40. Trebotic LK, Klimek P, Thomas A et al (2015) Circulating betatrophin is strongly increased in pregnancy and gestational diabetes mellitus. PLoS One 10:e0136701 